\title{
Improving management of gout in primary care using a customised electronic records template
}

Keith Moffat, Duncan McNab

NES

\begin{abstract} to improve the care of patients being treated for gout. recorded.

An audit was performed to measure and improve the following criteria:

- Monitoring of SUA levels in the past 12 months

- Titration of urate lowering therapy to bring the SUA below target level

- Lifestyle advice in the past 12 months
\end{abstract}

It is known that the management of chronic gout in relation to serum uric acid (SUA) monitoring, allopurinol dosing, and lifestyle advice is often sub-optimal in primary care.[1] A quality improvement project in the form of a criterion based audit was carried out in an urban general practice

Baseline searching of EMIS confirmed that management of patients with gout who were taking allopurinol was not in line with current guidance. 51(40\%) had a SUA checked in the past 12 months, 88(25\%) had a SUA below target level, and gout lifestyle advice was not being

An audit standard of $60 \%$ achievement at 2 months and $80 \%$ achievement at 4 months was set.

The intervention consisted of a custom electronic template within EMIS which allowed guidance of gout management to be displayed and for data to be entered. All members of the team including GPs and administrative staff were educated regarding the intervention. This resulted in a sustained improvement over a 6 month period in all 3 components of the audit with $112(84 \%)$ having a SUA level checked, $79(51 \%)$ having a SUA below target level and 76(57\%) receiving lifestyle advice. Although the improvement did not reach the audit standard in 2 of the criteria it would be expected that outcomes would continue given the systems changes which have been made.

\section{Problem}

The management of gout in the primary care setting is often suboptimal[1]. It was felt within my practice that guidance was not being followed in relation to the management of patients with gout. There was no recall system in place for patients on urate lowering therapy (ULT) and clinicians felt that blood monitoring of serum uric acid (SUA) levels in patients taking allopurinol and adjustment of drug dose thereafter was rarely performed. After starting allopurinol for the treatment of recurrent gout, patients were not routinely advised to have their SUA rechecked. As such the dose of allopurinol tended to remain static as opposed to being titrated according to SUA.

\section{Background}

This quality improvement project took place within London Road Medical Practice in Kilmarnock, Ayrshire, Scotland. It is a training practice in an urban area which serves a patient population of 12,722
It is known that the management of gout in relation to SUA monitoring, allopurinol dosing, and lifestyle advice is generally suboptimal in primary care.[1]

The British Society of Rheumatology guidelines 2007 provide useful guidance for the management of gout.[2] There have been more recent guidelines produced by the American College of Rheumatology in 2012 with the main difference in the latter being that allopurinol can be started during an acute flare of gout.[3, 4]

Several important best practice points were found within the guidelines. These included that patients with recurrent gout on ULT should have their SUA measured and should have lifestyle advice. SUA should be measured 2-5 weeks after dose adjustment of allopurinol. The target for SUA is less than $300 \mu \mathrm{mol} / \mathrm{l}$.

\section{Baseline measurement}

EMIS web[5], a primary care clinical system was used to conduct all of the relevant searches of patients within the practice. 
Read codes (N023, C34, 1443) identified those patients with the relevant diagnoses. This was combined with a search for those who were also issued allopurinol in the last six months.

This gave a baseline population of patients with gout taking allopurinol of 126 . Of these patients:

- $41 \%$ of patients had a SUA checked in the past 12 months from date of search.

- $88 \%$ of patient's most recent SUA was greater than $300 \mu \mathrm{mol} / \mathrm{L}$.

A new EMIS code was created specifically for the recording of "gout lifestyle advice". For this reason $0 \%$ of patients had this. There was no equivalent code being recorded for lifestyle advice being given to patients with gout.

See supplementary file: ds4683.pdf - "Results Table"

\section{Design}

A criterion based audit was performed. The mainstay of the intervention were three criteria which consisted of the following elements.

Patients taking allopurinol for gout should have:

- Lifestyle advice in the last 12 months?

- SUA measured in the last 12 months?

- SUA below target $(<300 \mu \mathrm{mol} / \mathrm{l})$ ?

The standard set for each of these components was $60 \%$ completion by 2 months and $80 \%$ by 4 months.

A custom template was designed and incorporated into EMIS. This allowed recording of lifestyle advice as well as providing guidance to the healthcare assistant regarding which bloods to take as well as advising the GP on guidelines regarding adjustment of allopurinol dose.

A letter was sent to all patients who had a SUA $>300 \mu \mathrm{mol} / \mathrm{l}$ and/or had not had a SUA checked in the past 12 calendar months. This also contained a patient information leaflet from the UK gout society giving lifestyle advice on gout.[6] The letter invited them to attend for blood tests and advised that their dose of allopurinol may be changed as a result of this. They were instructed to make an appointment with the healthcare assistant for this. The healthcare assistant was educated about the project and shown the EMIS template. They were advised to take bloods for U\&Es and SUA. The patient was instructed to phone for their results.

The GP who received the results would look at their current dose of allopurinol and decide whether a change was necessary. They would then arrange repeat SUA testing as appropriate.

Another subset of patients were those who were already known to have a raised SUA. The GPs were emailed an excel spreadsheet with a list of their patients for whom this was the case. They then decided on any changes of allopurinol dose and advised one of the administrative staff who actioned this and sent a letter to the patient. This also was to contain a patient information leaflet.

A 12 monthly search was set up on EMIS web. This is the first time that this has been done in the practice and a procedure for checking this and other automated searches is to be formalised. At present the practice development GP will have this responsibility. This search will also be of benefit in that it will follow those patients who have recently had an SUA level $<300 \mu \mathrm{mol} / \mathrm{l}$ but which in the future rises above threshold.

\section{Strategy}

A 'Plan, Do, Study, Act' model was used throughout the quality improvement process. The following summarises this.

PDSA 1

The first PDSA cycle planned to educate staff regarding the project and intervention and to implement the intervention. This involved a meeting with all of the team at a practice meeting. This included GPs, administrative staff, and healthcare assistants. An exce spreadsheet was sent to all GPs with details of patients with SUA $>300 \mu \mathrm{mol} / \mathrm{l}$ to be returned to the administrative manager to be actioned. A custom EMIS template was designed and installed on EMIS for data entry.

The outcome of this was that few GPs returned the excel spreadsheets to be actioned. Some returned handwritten notes to myself which were sometimes not legible. Of note the administrative aspect was more successful with patients who had no SUA in the past 12 months receiving letters and making appointments appropriately.

The plan for the next cycle would mainly focus on GPs completing and returning the excel spreadsheets.

PDSA 2

I sent an email reiterating the original meeting and reminding of the instructions for completing the spreadsheet. I also discussed this at a further practice meeting.

Following the 2nd PDSA cycle all GPs had reviewed the electronic record of their allocated patients and had implemented an appropriate plan. Unfortunately the letters that were sent out actioning these plans did not include the patient information leaflet. It was decided to give these to patients as they attended for blood tests on an ongoing basis. The patient information leaflet was uploaded to docman (a file management system) for this purpose.

PDSA 3

It became apparent from the 3rd data collection that opportunistic patient education was either not being done or wasn't being recorded within the electronic template. 
As a practice we will discuss whether to send out letters to all patients who were originally missed or continue with opportunistic education with further administration staff education. We will also look to add a function within the template would mean it would not be possible to complete it unless lifestyle advice had been given.

\section{Post-measurement}

Data for the care bundle was collected over a six month period. Once at the beginning of the project in May 2014, after the 1st PDSA cycle in July 2014 and again following the 2nd PDSA cycle in November 2014 (see table 1 in appendix).

- SUA measured in the last 12 months

Data following the final PDSA cycle showed a sustained increase in the number of patients who had a SUA measured in the last 12 months. The data points were 51 (40.4\%), 103 (81.7\%), and 112 (84.2\%). See run chart on appendix 1 figure 1.

$$
\text { - SUA<300 } \mu \mathrm{mol} / \mathrm{l}
$$

Data showed a sustained improvement in the percentage of those patients with SUA<300 $\mu \mathrm{mol} / /$. The data points were $88(25.5 \%)$, $57(33.1 \%)$, and $79(51.8 \%)$. See run chart on appendix 1 figure 2 . NB: the improvement in percentages despite less absolute number of patients was partly due to more people having SUA measured as part of the intervention

- Lifestyle advice

No patients had lifestyle advice for gout at the outset as discussed in the design section. The percentage increased when letters were sent to all patients who were invited to have their bloods checked $75(59.5 \%)$ and decreased after the second PDSA cycle 76(57.1\%) due to only one more person having lifestyle advice opportunistically and the gout population increasing in number.

This shows global improvement although in two of the criteria the audit standard was not met.

See supplementary file: ds4682.pdf - "Appendix 1 Run Charts"

\section{Lessons and limitations}

This project has shown that sustained improvements in the management of chronic gout is possible using a multi-criteria audit and with a global approach involving all of the members of the team.

This project was much more difficult than I expected it would be in terms of proactively managing the data collection from colleagues. It showed the importance of the whole team being involved so that when the project leader is not present, progress continues.

In terms of limitations the main one is that the results have not been bundled. The intention was for the intervention to be a care bundle.
However due to the fact data was not collected for all of the criteria together it was not possible to find out how many patients had all of the parts of the intervention.

A further limitation is the fact that now only an annual recall system is in place. This means that patients with gout newly started on ULT will not necessarily be managed in the same way as other patients until they reach annual recall. A way to improve this would be to have the gout template open up when patients are first prescribed ULT. This will be further investigated by the practice development GP.

Unfortunately the lifestyle advice aspect of the project was the least successful in part due to patient information leaflets not being sent out to those with raised SUA levels. In terms of systems change this is least important as this involved an oversight. This could have been improvement through the use of a protocol for reminding staff.

The audit will be run on a quarterly basis as part of a larger practice based drive for quality improvement to ensure sustainability. The practice development GP will be overseeing this but all members of the team are involved including the administration manager, practice manager, and healthcare assistants.

\section{Conclusion}

This project showed a sustained improvement in the number of patients having SUA levels checked and SUA levels under target levels. The management of chronic gout can be improved with criterion based audit, communication throughout the team, and changes in practice systems. The outcomes would be expected to be generalisable to other practice settings.

\section{References}

1. Jeyaruban A, Larkins S, Soden M. Management of gout in general practice-a systematic review. Clinical rheumatology 2014.

2. Jordan KM, Cameron JS, Snaith M, Zhang W, Doherty M, Seckl J, et al. British Society for Rheumatology and British Health Professionals in Rheumatology guideline for the management of gout. Rheumatology (Oxford, England). 2007;46(8):1372-4

3. Khanna D, Fitzgerald JD, Khanna PP, Bae S, Singh MK, Neogi T, et al. 2012 American College of Rheumatology guidelines for management of gout. Part 1: systematic nonpharmacologic and pharmacologic therapeutic approaches to hyperuricemia. Arthritis care Res 2012;64(10):1431-46.

4. Khanna D, Khanna PP, Fitzgerald JD, Singh MK, Bae S, Neogi T, et al. 2012 American College of Rheumatology guidelines for management of gout. Part 2: therapy and antiinflammatory prophylaxis of acute gouty arthritis. Arthritis care Res 2012;64(10):1447-61.

5. EMIS Web 2014 [10/03/2015]. Available from: http://www.emis-online.com/emis-web.

6. Society UG. All about gout and diet UK Gout Society 


\section{BMJ Quality Improvement Reports}

website [cited 2014 28/12/2014]. Available from: http://www.ukgoutsociety.org/PDFs/goutsocietyallaboutgoutanddiet-0113.pdf.

\section{Declaration of interests}

Nothing to declare.

\section{Acknowledgements}

Dr Stewart Morton London Road Medical Practice (LRMP)

Kilmarnock

Jennifer Allardice LRMP

Linda Caldwell LRMP

\section{Ethical approval}

Ethical approval was not sought as this QI project sought to improve compliance with current guidelines and study participants were not randomised to different processes of care.

Ethical approval was not required for this project as per the Medical Research Council Health Research Authority research decision tool guidance. 